\title{
Sporadic and intense accretion in a 1 Myr-old brown dwarf candidate
}

\author{
D. Nguyen-Thanh ${ }^{1,2,3}$, N. Phan-Bao ${ }^{1,2}$, S. J. Murphy ${ }^{4,5}$, and M. S. Bessell ${ }^{5}$ \\ ${ }^{1}$ Department of Physics, International University, Ho Chi Minh City, Vietnam \\ e-mail: pbngoc@hcmiu.edu.vn \\ 2 Vietnam National University, Ho Chi Minh City, Vietnam \\ ${ }^{3}$ Faculty of Physics and Engineering Physics, University of Science, Ho Chi Minh City, Vietnam \\ ${ }^{4}$ School of Science, University of New South Wales Canberra, ACT 2600, Australia \\ ${ }^{5}$ Research School of Astronomy and Astrophysics, Australian National University, Cotter Rd, Weston, ACT 2611, Australia
}

Received 30 September 2019 / Accepted 8 January 2020

\begin{abstract}
Context. Studying the accretion process in very low-mass objects has important implications for understanding their formation mechanism. Many nearby late-M dwarfs that have previously been identified in the field are in fact young brown dwarf members of nearby young associations. Some of them are still accreting. They are therefore excellent targets for further studies of the accretion process in the very low-mass regime at different stages.

Aims. We aim to search for accreting young brown dwarf candidates in a sample of 85 nearby late-M dwarfs.

Methods. Using photometric data from DENIS, 2MASS, and WISE, we constructed the spectral energy distribution of the late$\mathrm{M}$ dwarfs based on BT-Settl models to detect infrared excesses. We then searched for lithium and $\mathrm{H} \alpha$ emission in candidates that exhibit infrared excesses to confirm their youth and the presence of accretion.

Results. Among the 85 late-M dwarfs, only DENIS-P J1538317-103850 (M5.5) shows strong infrared excesses in WISE bands. The detection of lithium absorption in the M5.5 dwarf and its Gaia trigonometric parallax indicate an age of $\sim 1$ Myr and a mass of $47 M_{J}$. The $\mathrm{H} \alpha$ emission line in the brown dwarf shows significant variability that indicates sporadic accretion. This 1 Myr-old brown dwarf also exhibits intense accretion bursts with accretion rates of up to $10^{-7.9} M_{\odot} \mathrm{yr}^{-1}$.

Conclusions. Our detection of sporadic accretion in one of the youngest brown dwarfs might imply that sporadic accretion at early stages could play an important role in the formation of brown dwarfs. Very low-mass cores would not be able to accrete enough material to become stars, and thus they end up as brown dwarfs.
\end{abstract}

Key words. techniques: spectroscopic - techniques: photometric - brown dwarfs - circumstellar matter - stars: low-mass

\section{Introduction}

Very low-mass (VLM) objects, with masses below $0.35 M_{\odot}$ or spectral types later than M3-M4, are expected to form like lowmass stars (e.g., see Luhman et al. 2007 and references therein). However, physical processes at different stages of their formation such as accretion are still poorly understood. VLM populations in nearby young associations at different ages, especially objects with detected circumstellar disks (e.g., Reiners 2009; Murphy et al. 2015, 2018; Boucher et al. 2016; Silverberg et al. 2016), are important resources for studying the formation mechanism of VLM objects as well as planets around them.

Typically, new candidate members of young associations have been identified using proper motions, spatial positions, distances, and color-magnitude diagrams (e.g., Murphy et al. 2015; Luhman et al. 2018). Their membership is then confirmed using spectral diagnostics of youth such as the Li I doublet line at $6708 \AA$, and the Na I doublet at $8183 \AA$ and $8199 \AA$.

Circumstellar disks around young stars are warm enough to produce detectable infrared (IR) excesses. Therefore, infrared photometry has also been used to identify new disk-bearing members (e.g., Avenhaus et al. 2012; Silverberg et al. 2016; Boucher et al. 2016). Avenhaus et al. (2012) searched for circumstellar disks around 85 nearby $M$ dwarfs and found no IR excesses in any of them. Boucher et al. (2016) discovered three
late-M dwarfs (M4.5-M9) and one L dwarf with candidate circumstellar (primordial or pre-transitional) disks from a sample of 1600 objects. They are new candidate members of TW Hya ( 10 Myr), Columba ( 42 Myr) and Tucana-Horologium ( $\sim 45 \mathrm{Myr})$. Silverberg et al. (2016) searched for new members of nearby young associations using Wide-field Infrared Survey Explorer (WISE) data among a sample of 1774 objects. The authors found only an M5 dwarf (WISE J080822.18-644357.3, hereinafter WISE0808-6443) with significant excesses at 12 and $22 \mu \mathrm{m}$. The excesses indicate that the $\mathrm{M}$ dwarf has a circumstellar disk. Based on the spatial coordinates, proper motion and photometric distance, they concluded that WISE0808-6443 is a debris disk-bearing 45 Myr-old candidate member of the Carina association. Murphy et al. (2018) measured the velocity width of the $\mathrm{H} \alpha$ emission line and proved that the M5 dwarf is in fact still accreting. The IR excesses and accretion activity imply that WISE0808-6443 likely hosts a primordial disk. However, the detection of weak millimeter emission and the lack of detectable CO emission recently reported in Flaherty et al. (2019) indicate that WISE0808-6443 hosts a debris disk.

New members of nearby associations have also been revealed serendipitously by spectroscopic observations of nearby lateM dwarf candidates in the field (e.g., Reiners 2009; Martín et al. 2010; Phan-Bao et al. 2017; Riedel et al. 2019 and references therein). DENIS-P J0041353-562112, which was initially 
identified as a nearby M7.5 dwarf (Phan-Bao et al. 2001; Phan-Bao \& Bessell 2006) turns out to be a young brown dwarf (BD) binary (M6.5+M9.0, Reiners 2009; Reiners et al. 2010) member of the Tucana-Horologium association. Surprisingly, the primary M6.5 dwarf component is still accreting. Martín et al. (2010) spectroscopically observed 78 ultracool dwarf candidates in the field and revealed seven new members of the Upper Scorpii OB association. Phan-Bao et al. (2017) found a new member of the $\beta$ Pic moving group and other young candidate members.

In this paper, we report our search for new disk-bearing late$M$ dwarfs from a sample of 85 nearby late-M dwarfs. Section 2 presents the sample and our search for IR excesses. In Sect. 3, we present the results of our search, detections of lithium and observations of $\mathrm{H} \alpha$ emission at different epochs in DENISP J1538317-103850 (hereinafter DENIS1538-1038). We then estimate the mass and age of the source. We also discuss sporadic accretion in DENIS1538-1038 and its implication for BD formation. Section 4 summarizes our results.

\section{Sample selection and a search for IR excesses}

We selected a sample of 85 nearby late-M dwarfs, which have previously been found, from the Deep Near Infrared Survey of the Southern Sky (DENIS) (Phan-Bao et al. 2001, 2003, 2006, 2017; Crifo et al. 2005; Phan-Bao \& Bessell 2006). The lateM dwarfs were originally detected over 5700 square degrees in the DENIS database at high Galactic latitude with $|b| \geq 30^{\circ}$ (Phan-Bao et al. 2001, 2003), photometric distances within 30 pc and the color range $2.0 \leq I-J \leq 3.0$ (M6-M8). They were spectroscopically observed to determine spectral types and distances (Crifo et al. 2005; Phan-Bao \& Bessell 2006). One of them is a triple system (Phan-Bao et al. 2006). The sample also included a few very nearby late-M dwarfs within $13 \mathrm{pc}$ (Phan-Bao et al. 2017). Totally, we have 85 late-M dwarfs with IR photometry available in the WISE database. These late-M dwarfs have spectral types ranging from M5.0 to M9.0. In our previous paper (Phan-Bao et al. 2017), we searched for the Li I $6708 \AA$ absorption line in 28 of these 85 late-M dwarfs and detected lithium in five of them. Therefore, we classified these five late-M dwarfs as VLM candidate members of young associations or the young field. This implies that the sample could contain more potential young VLM objects, especially disk-bearing objects.

We therefore constructed the spectral energy distribution (SED) of all 85 late-M dwarfs to search for IR excesses. We used photometric data with $I$-band magnitudes from DENIS, $J, H$, and $K_{\mathrm{S}}$-band magnitudes from the Two Micron All Sky Survey (2MASS) and mid-infrared $W 1, W 2, W 3$, and $W 4$-band magnitudes from WISE (Cutri et al. 2014). Zero magnitude flux values for DENIS (Fouqué et al. 2000), 2MASS (Cohen et al. 2003), and WISE (Wright et al. 2010) were used to convert from magnitudes to fluxes. BT-Settl model atmospheres (Allard et al. 2013) with solar metallicity and surface gravities ranging from $\log g=3.0$ to 5.5 were used to find the best fits of these models to the observed photometric data. Trigonometric distances were used if available in Gaia. We then only selected candidates with observed infrared fluxes more than $3 \sigma$ above the model fluxes (where $\sigma$ is the error on the observed fluxes). There are 11 candidates in total that match this criterion. These candidates were further examined visually in the co-added images from all four WISE bands ${ }^{1}$. The fluxes of a source measured in $W 3$ and $W 4$ bands are reliable if the source is clearly detected in the images

1 https://irsa.ipac.caltech.edu/applications/wise/ of these bands. We also examined the possibility that $W 3$ and $W 4$ fluxes are contaminated by background objects using $\mathrm{SERC}^{2}$, DENIS $^{3}$ and 2 MASS $^{4}$ images.

\section{Results and discussion}

Among the 85 late-M dwarfs, we only detected IR excesses in DENIS1538-1038, indicating that the source hosts an accretion disk (see Sect. 3.6 for further details). Our spectroscopic follow-up revealed the presence of lithium in DENIS1538-1038, confirming its youth. We then used the absolute magnitude versus color diagram to determine the age and the mass of DENIS1538-1038. To estimate accretion rate, we measured the velocity width of the $\mathrm{H} \alpha$ line profile at $10 \%$ of the peak flux. We also observed DENIS1538-1038 at different epochs to confirm the sporadicity of the accretion process in the source.

\subsection{IR excesses in DENIS1538-1038}

DENIS1538-1038 $\quad\left(\alpha_{\mathrm{J} 2000}=15^{\mathrm{h}} 38^{\mathrm{m}} 31.70^{\mathrm{s}}, \quad \delta_{\mathrm{J} 2000}=\right.$ $\left.-10^{\circ} 38^{\prime} 50.6^{\prime \prime}\right)$ shows significant IR excesses in all four WISE bands (Fig. 1) that are above 3,10, 27 and $9 \sigma$ for bands $W 1, W 2, W 3$, and $W 4$, respectively. Phan-Bao et al. (2003) identified DENIS1538-1038 as a low-proper motion M dwarf $\left(\mu<0.1^{\prime \prime} \mathrm{yr}^{-1}\right)$. DENIS1538-1038 has a spectral type of M5.0 and a spectroscopic distance of 31.7 pc (Crifo et al. 2005). Figure 2 shows the SED of DENIS1538-1038. The SEDs of the 84 remaining $\mathrm{M}$ dwarfs are shown in Appendix A.

During the course of examining the WISE images of DENIS1538-1038, we serendipitously identified DENIS-P $\mathrm{J} 1538316-103900 \quad\left(\alpha_{\mathrm{J} 2000}=15^{\mathrm{h}} 38^{\mathrm{m}} 31.62^{\mathrm{s}}, \quad \delta_{\mathrm{J} 2000}=\right.$ $-10^{\circ} 39^{\prime} 00.4^{\prime \prime}$, hereinafter DENIS1538-1039) at a separation of $\sim 10^{\prime \prime}$ from DENIS1538-1038. Both objects have proper motions and trigonometric parallaxes measured by Gaia. DENIS1538-1038 has a low-proper motion as measured in

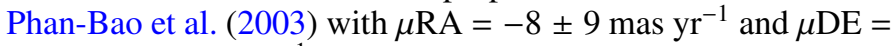
$-18 \pm 9$ mas $\mathrm{yr}^{-1}$. These proper motion values are in agreement with the Gaia measurements, $\mu \mathrm{RA}=-16.728 \pm$

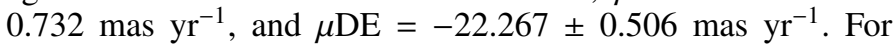
DENIS1538-1039, the dwarf also has a low-proper motion from Gaia with $\mu \mathrm{RA}=-14.622 \pm 0.598$ mas $\mathrm{yr}^{-1}$ and $\mu \mathrm{DE}=$

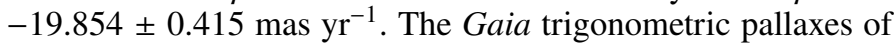
DENIS1538-1038 and DENIS1538-1039 are 9.2849 \pm 0.3494 mas (or $107.7 \pm 4.1 \mathrm{pc}$ ) and $7.8228 \pm 0.2936 \mathrm{mas}$ (or $127.8 \pm 4.8 \mathrm{pc}$ ), respectively. Their proper motions and distances suggest that they might belong to a nearby young association. A DENIS color $I-J=1.5$ (Table 1) suggests that DENIS1538-1039 has a spectral type of $\sim \mathrm{M} 4$ (Bessell 1991). We also searched for IR excesses in DENIS1538-1039, however, its SED shows no evidence of a circumstellar disk (Fig. 3).

We note that, with a separation of $10^{\prime \prime}$, DENIS1538-1038 and DENIS1538-1039 are spatially resolved in the WISE $W 1$, $W 2$, and $W 3$ images (see Fig. 1) with corresponding angular resolutions of 6.1", 6.4", and 6.5" (Wright et al. 2010). However, the resolution of the $W 4$ image of $12^{\prime \prime}$ is not capable of resolving these two sources. DENIS1538-1039 could thus contribute its flux to the IR excess in the W4 band of DENIS1538-1038 (see Sect. 3.6 for further discussion). In order to confirm the presence of accretion and the young nature of these two $M$ dwarfs, we

\footnotetext{
2 https://archive.stsci.edu/cgi-bin/dss_plate_finder

3 http://cds.u-strasbg.fr/denis.html

4 https://irsa.ipac.caltech.edu/applications/2MASS/IM/ interactive.html
} 

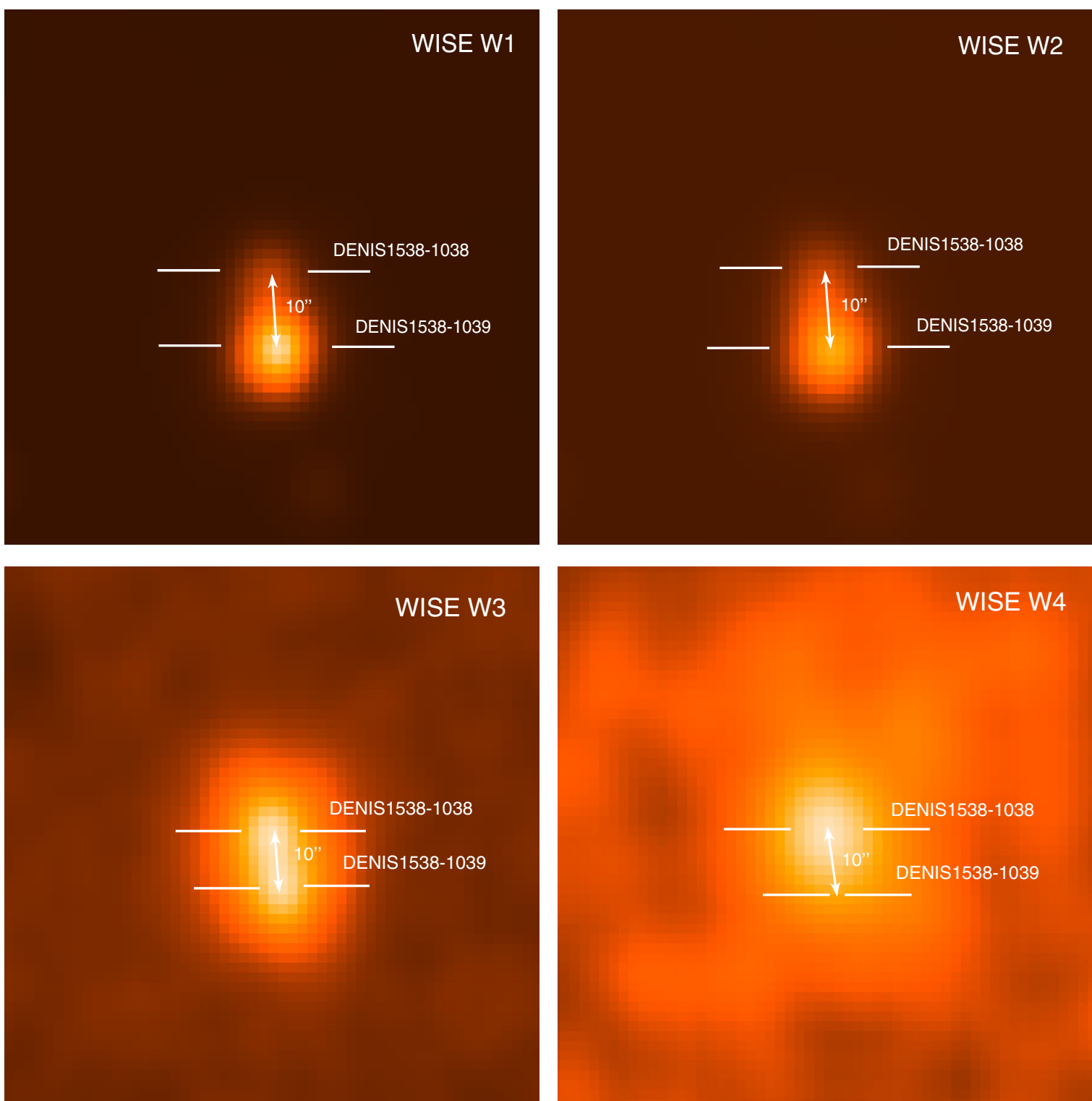

Fig. 1. $W 1, W 2, W 3$, and $W 4$ WISE images for DENIS1538-1038 and DENIS1538-1039 (see Sect. 3.1 for further discussion). DENIS1538-1039 is not visible in the W4 image. The bars indicate the positions of DENIS1538-1038 and DENIS1538-1039. A separation of 10" between the two objects is also indicated. The charts are $\sim 1.25 \times \sim 1.25 \mathrm{arcmin}^{2}$. North is up, and east is to the left.

observed the $\mathrm{H} \alpha$ emission line and searched for $\mathrm{Li}$ I absorption at $6708 \AA$.

\subsection{Spectroscopic follow-up of DENIS1538-1038 and DENIS1538-1039}

We observed the two M dwarfs in Apr., May, and Aug. 2017, and Jul. 2018, with the Wide Field Spectrograph (WiFeS, Dopita et al. 2007) on the ANU $2.3 \mathrm{~m}$ telescope at Siding Spring Observatory. We used the $\mathrm{R} 7000$ grating over a wavelength range of 5280-7050 A, providing a spectral resolution of $R \approx 7000$. The signal-to-noise ratios $(\mathrm{S} / \mathrm{Ns})$ of spectra are in the range of $10-21$. Table 2 lists the observing dates of the two sources. We additionally observed them on Mar. 22, 2017 with the R3000 grating, $R \approx 3000$ over 5660-9640 $\AA$, to estimate the spectral type of the newly identified M dwarf DENIS1538-1039.

We then used FIGARO (Shortridge et al. 2004) for data reduction. Only the R7000 spectra were flux-calibrated. Each night, two flux standards, such as EG 21, EG 131, LTT4364, and L745-46A were observed. The R7000 spectra were not corrected for telluric absorption. For the R3000 spectra, smooth spectrum stars were additionally observed to remove the telluric lines (see Bessell 1999). A NeAr arc was used for wavelength calibration.

Figure 4 shows the spectra of DENIS1538-1039 and DENIS1538-1038. We estimated the spectral type of DENIS1538-1039 as in Phan-Bao et al. (2017). The adopted spectral type is an average value calculated from three indices $\mathrm{VOa}, \mathrm{TiO} 5$ and PC3. Our calculation gives a spectral type of M3.5 \pm 0.5 for DENIS1538-1039. With a new R3000 spectrum, we also obtained a spectral type of M5.5 \pm 0.5 for DENIS1538-1038, which is in agreement with the spectral type M5.0 \pm 0.5 determined in Crifo et al. (2005). In this paper, we adopt the spectral type of M5.5. We note that the DENIS colors of DENIS1538-1039 $(I-J=1.5)$ and DENIS1538-1038 $(I-J=2.18$, Phan-Bao et al. 2003) are also consistent with the intrinsic colors of young $(5-30 \mathrm{Myr}) \mathrm{M} 3.5(I-J \sim 1.5)$ and 
Table 1. WISE photometry data of DENIS1538-1038 and DENIS1538-1039.

\begin{tabular}{lcccccccc}
\hline \hline Name & $\begin{array}{c}I_{\text {DENIS }} \\
(\mathrm{mag})\end{array}$ & $\begin{array}{c}J_{2 \text { MASS }} \\
(\mathrm{mag})\end{array}$ & $\begin{array}{c}H_{2 \mathrm{MASS}} \\
(\mathrm{mag})\end{array}$ & $\begin{array}{c}K_{2 \mathrm{MASS}} \\
(\mathrm{mag})\end{array}$ & $\begin{array}{c}W 1 \\
(\mathrm{mag})\end{array}$ & $\begin{array}{c}W 2 \\
(\mathrm{mag})\end{array}$ & $\begin{array}{c}W 3 \\
(\mathrm{mag})\end{array}$ & $\begin{array}{c}W 4 \\
(\mathrm{mag})\end{array}$ \\
\hline $\mathrm{D*} 1538-1038$ & $14.36 \pm 0.23$ & $12.029 \pm 0.026$ & $11.447 \pm 0.030$ & $11.096 \pm 0.023$ & $10.738 \pm 0.034$ & $10.240 \pm 0.034$ & $8.758 \pm 0.035$ & $6.924 \pm 0.110$ \\
D*1538-1039 & $11.63 \pm 0.21$ & $10.007 \pm 0.024$ & $9.349 \pm 0.026$ & $9.111 \pm 0.023$ & $8.992 \pm 0.022$ & $8.874 \pm 0.020$ & $8.759 \pm 0.031$ & $>8.101$ \\
\hline
\end{tabular}

Notes. D*: DENIS.

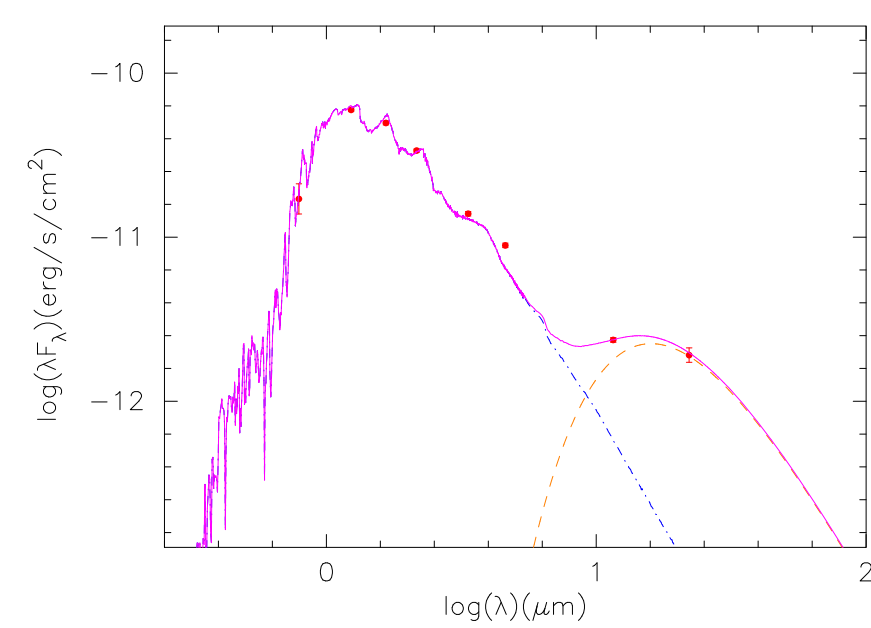

Fig. 2. SED for DENIS1538-1038. The blue dash-dotted line shows the BT-Settl model atmosphere $\left(T_{\star}=2800 \mathrm{~K}, \log g=3.5\right)$. The disk blackbody model $\left(T_{\text {disk }}=224 \mathrm{~K}\right)$ is indicated by the orange dashed line. Our best fit to the photometric data is shown by the magenta solid line.

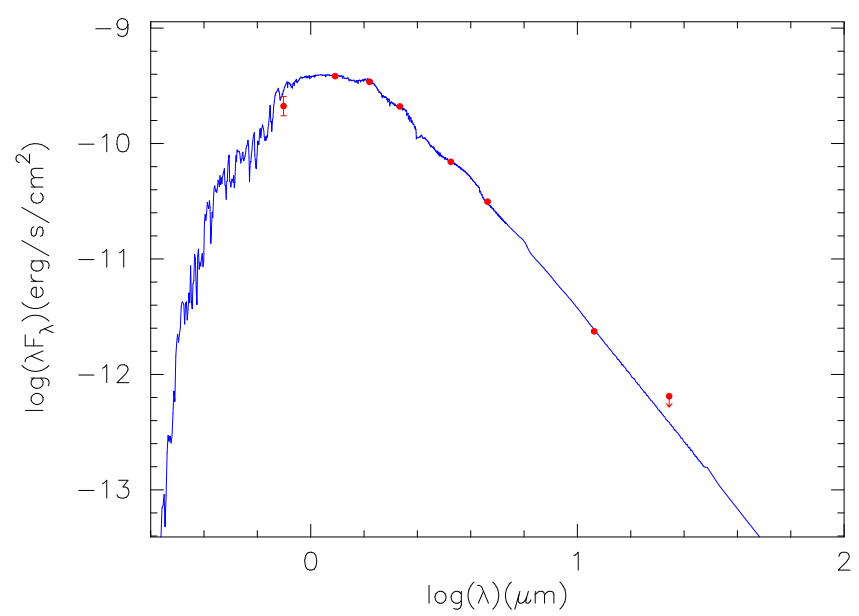

Fig. 3. SED for DENIS1538-1039. The blue solid line shows the BTSettl model atmosphere.

M5.5 (I-J 2.1) dwarfs (e.g., Pecaut \& Mamajek 2013). This implies that the reddening effect is not significant for the two $\mathrm{M}$ dwarfs. Therefore, we do not consider the de-reddening of SEDs in this paper.

We used the IRAF task splot to measure equivalent widths (EWs) of the Li I, H $\alpha$, and $\mathrm{He} \mathrm{I}$ at $5876 \AA$ and $6678 \AA$, Na I D1 (5895.9 A) D2 (5889.9 $)$, and [O I] $6300 \AA$ lines. The EW uncertainties were estimated by measuring EWs with different possible continuum levels and the noise around the regions of interest. In the cases of nondetection of the lines, upper limits were given by measuring the noise. Table 2 lists our measurements.

\subsection{Lithium detection}

The Li I doublet line at $6708 \AA$ is clearly detected in both DENIS1538-1038 and DENIS1538-1039 (Fig. 5). These detections confirm that the two $\mathrm{M}$ dwarfs are young (see Basri 2000 and references therein). Our EW measurements of the gravity-sensitive indicator $\mathrm{Na} I(8183 / 8199 \AA)$ as described in Martín et al. (2010) from the R3000 spectra are $4.6 \pm 0.9 \AA$ and $4.6 \pm 1.9 \AA$ for DENIS1538-1038 and DENIS1538-1039, respectively. The Na I EW of DENIS1538-1038 is in good agreement with our previous measurement of $4.3 \pm 0.9$ (Phan-Bao et al. 2017). We note that we did not detect lithium in DENIS1538-1038 in the previous observation (Phan-Bao et al. 2017) due to a low $S / N$. Here, the $S / N$ in the lithium region of the spectra is about 15 , significantly higher than the previous one of $\sim 7$ (Phan-Bao et al. 2017). The lithium line is thus revealed in all spectra (Table 2).

\subsection{Age and mass determination}

Using the Gaia distance of $107.7 \pm 4.1 \mathrm{pc}$ and the DENIS $J$-band apparent magnitude $J=12.18 \pm 0.23$, we derived an absolute magnitude $M_{J}=7.02 \pm 0.31$ for DENIS1538-1038. According to the BT-Settl atmosphere models for the DENIS photometric system (Allard et al. 2013), the $J$-band absolute magnitude of 7.02 and the color $I-J=2.18$ of the M5.5 dwarf correspond to an age of $\sim 1$ Myr and a mass of $\sim 47 M_{J}$ (Fig. 6). Our estimated mass indicates that DENIS1538-1038 is a young BD. If the error of the $I-J$ color is taken into account, we derive upper limits of $5 \mathrm{Myr}$ and $90 M_{J}$ for the age and the mass of the source, respectively.

For the case of DENIS1538-1039, its Gaia distance of $127.8 \pm 4.8$ and magnitude $J=10.13 \pm 0.21$ give $M_{J}=$ $4.60 \pm 0.29$. The derived $J$-band absolute magnitude and the color $I-J=1.5$ give an age of $\sim 2 \mathrm{Myr}$ and a mass of $\sim 0.33 \mathrm{M}_{\odot}$. The error of the $I-J$ color and the presence of lithium (Fig. 6) in the M3.5 dwarf place upper limits of $4 \mathrm{Myr}$ and $0.48 M_{\odot}$ on its age and mass, respectively.

The detections of lithium in both DENIS1538-1038 and DENIS1538-1039 clearly indicate the youth of the two sources. Therefore, they are candidate members of nearby young associations. To calculate the membership probability of these two $\mathrm{M}$ dwarfs in nearby associations, we used the Bayesian analysis tool BANYAN $\Sigma^{5}$ (see Gagné et al. 2018). With the parallaxes and proper motions from Gaia, we found that DENIS 1538-1038 has a membership probability of $31.4 \%$ for Upper Scorpius and $68.6 \%$ for the young field. For DENIS1538-1039, we found a membership probability of $14.3 \%$ for Upper Scorpius and $85.7 \%$ for the young field. However, the estimated age ranges of 1-5 Myr for DENIS1538-1038, and 1-4 Myr for DENIS1538-1039, indicate that they are more likely to

\footnotetext{
5 http://www.exoplanetes. umontreal.ca/banyan/ banyansigma.php
} 
Table 2. Observational results of DENIS1538-1038 and DENIS1538-1039.

\begin{tabular}{|c|c|c|c|c|c|c|c|c|c|c|}
\hline Name & $\begin{array}{l}\text { UT observing } \\
\text { date } \\
(2) \\
\end{array}$ & (3) & (4) & $\begin{array}{l}v_{10}(\mathrm{H} \alpha) \\
\left(\mathrm{km} \mathrm{s}^{-1}\right) \\
(5)\end{array}$ & $\begin{array}{l}\log (\dot{M}) \\
\left(M_{\odot} \mathrm{yr}^{-1}\right) \\
(6)\end{array}$ & $\begin{array}{l}\text { He I } \\
5876 \AA \\
(7) \\
\end{array}$ & $\begin{array}{l}\text { He I } \\
6678 \AA \\
(8)\end{array}$ & $\begin{array}{l}\text { Na I D1 } \\
5895.9 \AA \\
(9) \\
\end{array}$ & $\begin{array}{l}\text { Na I D2 } \\
5889.9 \AA \\
(10)\end{array}$ & $\begin{array}{l}{[\mathrm{O} I]} \\
6300 \AA \\
(11) \\
\end{array}$ \\
\hline \multirow[t]{5}{*}{ D*1538-1038 } & 19 Apr. 2017 & $0.24 \pm 0.02$ & $-60 \pm 2$ & $495 \pm 6$ & $-8.1 \pm 0.1$ & $-8.9 \pm 0.6$ & $-2.4 \pm 0.1$ & $-0.8 \pm 0.1$ & $-1.1 \pm 0.1$ & $-1.3 \pm 0.1$ \\
\hline & 4 May 2017 & $0.38 \pm 0.02$ & $-50 \pm 3$ & $511 \pm 11$ & $-7.9 \pm 0.1$ & $-5.8 \pm 0.4$ & $-1.6 \pm 0.1$ & $-0.6 \pm 0.2$ & $-0.7 \pm 0.2$ & $-1.2 \pm 0.1$ \\
\hline & 5 May 2017 & $0.22 \pm 0.01$ & $-49 \pm 1$ & $489 \pm 7$ & $-8.1 \pm 0.1$ & $-7.7 \pm 0.2$ & $-2.9 \pm 0.2$ & $-0.6 \pm 0.1$ & $-0.6 \pm 0.1$ & $-1.2 \pm 0.1$ \\
\hline & 12 Aug. 2017 & $0.47 \pm 0.02$ & $-25 \pm 2$ & $388 \pm 12$ & $-9.1 \pm 0.1$ & $-4.1 \pm 0.2$ & $-0.9 \pm 0.2$ & $>-0.2$ & $>-0.1$ & $-0.8 \pm 0.1$ \\
\hline & 17 Jul. 2018 & $0.41 \pm 0.02$ & $-13 \pm 1$ & $243 \pm 16$ & $-10.5 \pm 0.2$ & $-2.0 \pm 0.2$ & $-0.3 \pm 0.1$ & $0.4 \pm 0.2$ & $0.9 \pm 0.2$ & $-0.7 \pm 0.1$ \\
\hline \multirow[t]{8}{*}{ D*1538-1039 } & 19 Apr. 2017 & $0.48 \pm 0.02$ & $-1.7 \pm 0.1$ & $148 \pm 9$ & & $>-0.2$ & $>-0.1$ & $2.0 \pm 0.2$ & $3.2 \pm 0.2$ & $>-0.1$ \\
\hline & 4 May 2017 & $0.50 \pm 0.02$ & $-2.3 \pm 0.1$ & $149 \pm 7$ & & $>-0.2$ & $>-0.1$ & $1.9 \pm 0.2$ & $2.8 \pm 0.2$ & $>-0.1$ \\
\hline & 5 May 2017 & $0.48 \pm 0.02$ & $-2.0 \pm 0.2$ & $149 \pm 9$ & & $>-0.2$ & $>-0.1$ & $1.9 \pm 0.2$ & $2.9 \pm 0.2$ & $>-0.1$ \\
\hline & 12 May 2017 & $0.49 \pm 0.02$ & $-1.9 \pm 0.1$ & $157 \pm 12$ & & $>-0.3$ & $>-0.1$ & $2.2 \pm 0.2$ & $2.4 \pm 0.2$ & $>-0.2$ \\
\hline & 12 Aug. 2017 & $0.46 \pm 0.03$ & $-1.9 \pm 0.1$ & $147 \pm 9$ & & $>-0.1$ & $>-0.1$ & $2.1 \pm 0.2$ & $2.9 \pm 0.2$ & $>-0.2$ \\
\hline & 28 Aug. 2017 & $0.48 \pm 0.02$ & $-1.8 \pm 0.1$ & $153 \pm 6$ & & $>-0.2$ & $>-0.1$ & $2.0 \pm 0.2$ & $2.3 \pm 0.2$ & $>-0.1$ \\
\hline & 29 Aug. 2017 & $0.45 \pm 0.03$ & $-2.0 \pm 0.1$ & $142 \pm 6$ & & $>-0.1$ & $>-0.1$ & $2.0 \pm 0.2$ & $2.7 \pm 0.2$ & $>-0.1$ \\
\hline & 17 Jul. 2018 & $0.47 \pm 0.02$ & $-1.4 \pm 0.1$ & $156 \pm 10$ & & $>-0.1$ & $>-0.1$ & $2.2 \pm 0.2$ & $2.4 \pm 0.2$ & $>-0.2$ \\
\hline
\end{tabular}

Notes. D*: DENIS. Columns 3, 4, 7-11: equivalent widths in Å. No accretion rate is estimated for DENIS1538-1039, as we classify the source as a nonaccretor with the current data.

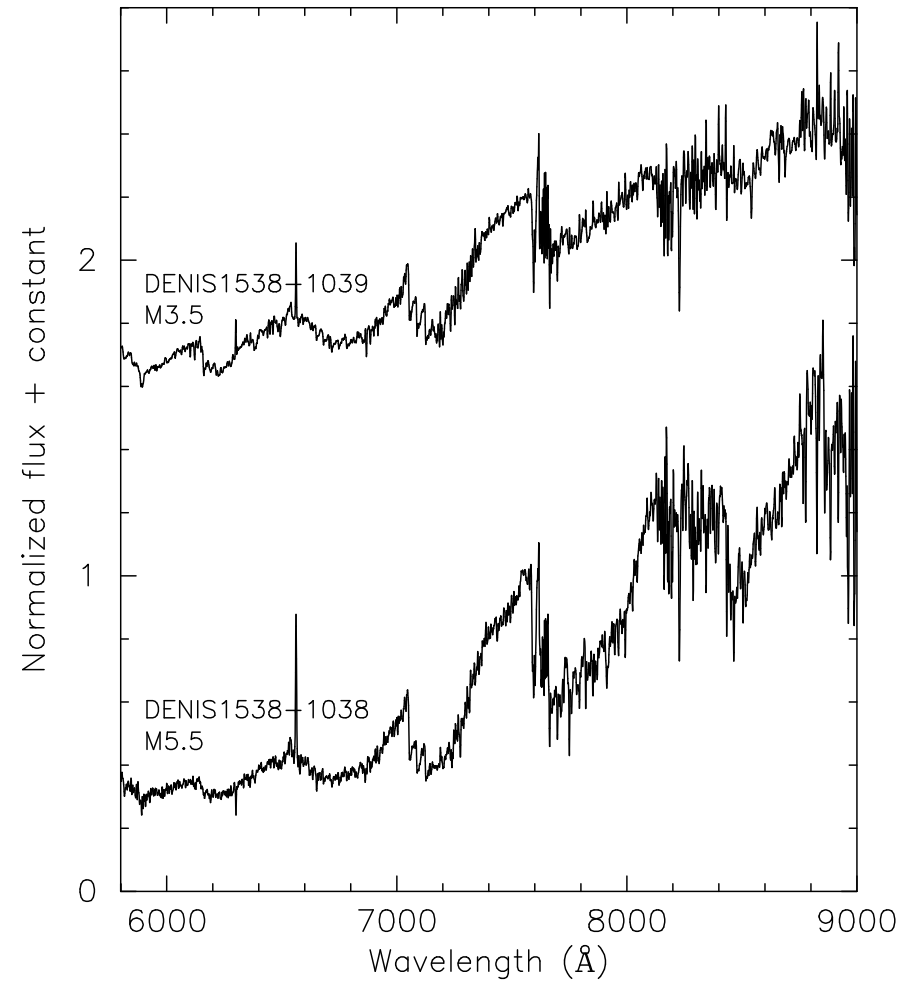

Fig. 4. R3000 spectra of DENIS1538-1039 and DENIS1538-1038. Spectral types adopted from spectral indices VOa, TiO5 and PC3 are also shown.

be members of young associations than young field dwarfs. BANYAN also returns predicted radial velocities for Upper Scorpius membership to be $-9.7 \pm 3.8$ and $-9.9 \pm 3.8 \mathrm{~km} \mathrm{~s}^{-1}$ for DENIS1538-1038 and DENIS1538-1039. At this point, we also measured radial velocities by cross-correlation against standards as described in detail in Murphy \& Lawson (2015). Our measured radial velocities of DENIS1538-1038 and DENIS1538-1039 range from $-4.5 \pm 1.2$ to $-14.9 \pm 1.6 \mathrm{~km} \mathrm{~s}^{-1}$ and from $-2.2 \pm 0.7$ to $-5.3 \pm 1.4 \mathrm{~km} \mathrm{~s}^{-1}$, respectively. The predicted values are in agreement with our measurements, further

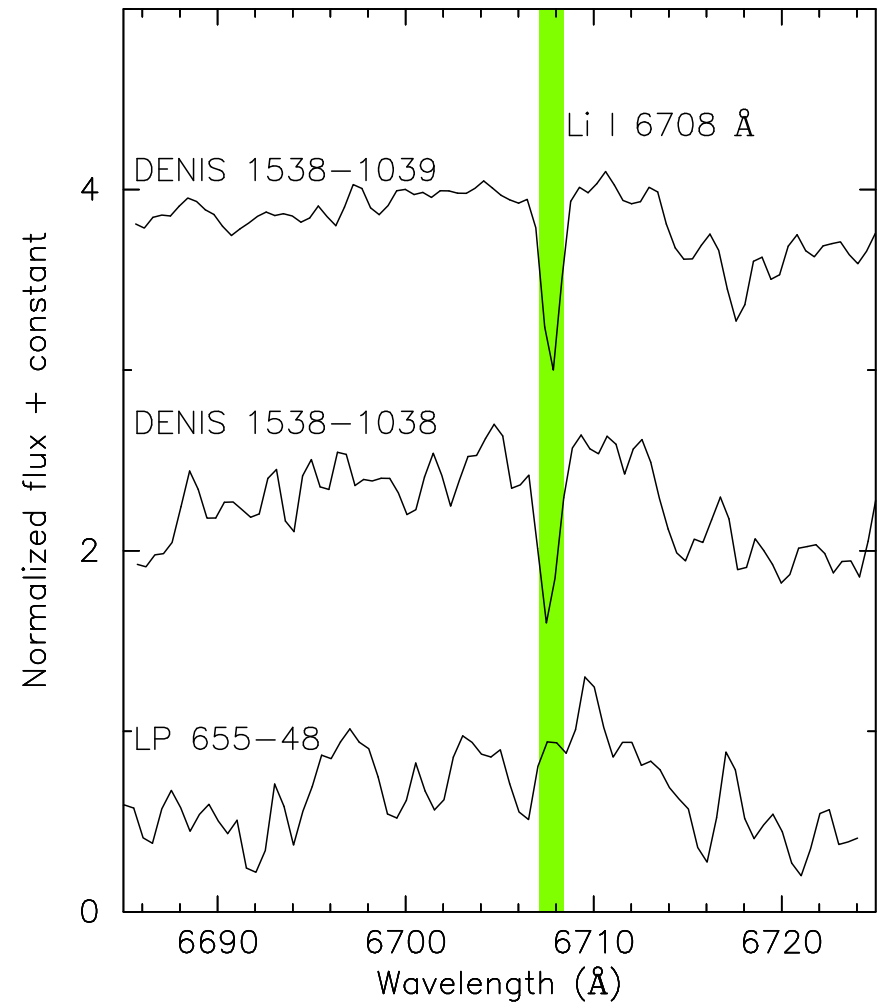

Fig. 5. R7000 spectra of DENIS1538-1039 and DENIS1538-1038 observed on Apr. 19, 2017. The spectrum of LP 655-48 with no lithium detection (Phan-Bao et al. 2017) is also plotted. The region of the Li I line (Pavlenko et al. 1995) is indicated.

supporting the membership of these two M dwarfs within Upper Scorpius. A detailed analysis of radial velocity measurements is to be given in a forthcoming paper. A possible scenario to explain the low Upper Scorpius membership probabilities of DENIS1538-1038 and DENIS1538-1039 is that the two $\mathrm{M}$ dwarfs are members of a new and very young population of 1-5 Myr stars in Upper Scorpius. The previous detection (Rizzuto et al. 2015) of young M dwarfs aged 2-5 Myr in Upper Scorpius supports our scenario. 


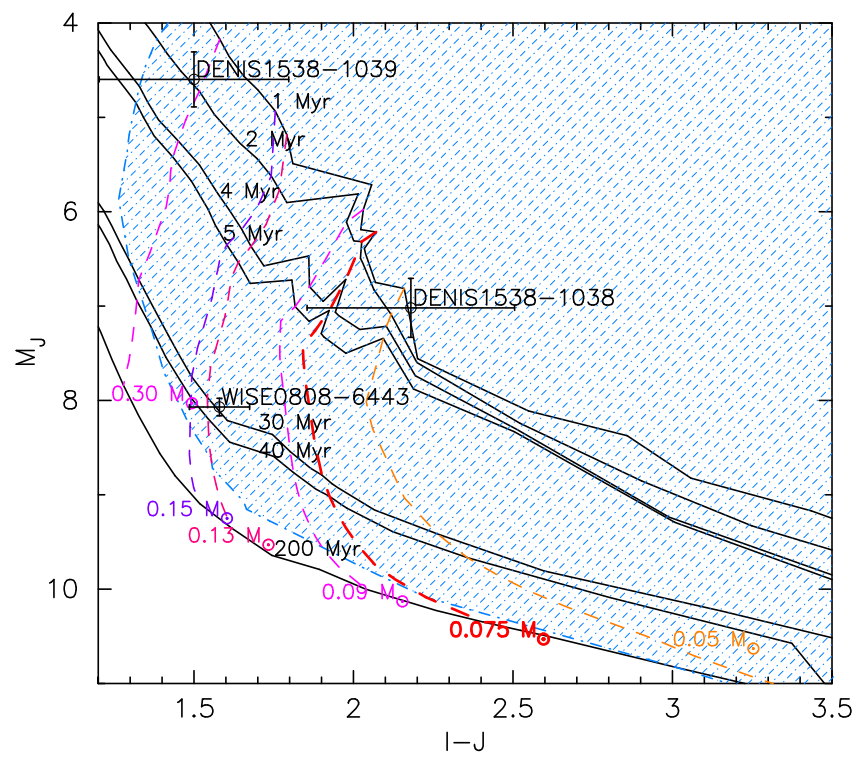

Fig. 6. $J$-band absolute magnitude versus color $I-J$ diagram for the accreting M5.5 dwarf (DENIS1538-1038) and the nonaccreting M3.5 one (DENIS1538-1039). Isochrones and mass tracks from the BT-Settl models (Allard et al. 2013) are shown. The blue hatched area indicates where lithium remains unburnt and detectable in the stellar photosphere. The 45 Myr-old accreting M5 dwarf WISE0808-6443 (Silverberg et al. 2016; Murphy et al. 2018) is also plotted. With a Gaia parallax of $9.8599 \pm 0.0551$ mas, the age of WISE0808-6443 is $\sim 30 \mathrm{Myr}$ and its mass is $\sim 0.12 M_{\odot}$.

\subsection{Sporadic accretion in DENIS1538-1038}

Among many indicators of accretion and outflow activities (e.g., see Herczeg \& Hillenbrand 2008; Rigliaco et al. 2012), we only detected $\mathrm{H} \alpha$, He I at $5876 \AA$ and $6678 \AA$, Na I D1 (5895.9 $\AA$ ) and D2 (5889.9 $\AA$ ), and [O I] $6300 \AA$ in the spectra of DENIS1538-1038 (Fig. 7). The strong H $\alpha$ emission implies that the observed IR excesses are from an accretion disk. To confirm the presence of accretion, we measured the velocity width of the $\mathrm{H} \alpha$ line profile at $10 \%$ of the peak flux (Fig. 8), which is listed as $v_{10}$ in Table 2. White \& Basri (2003) proposed $v_{10}>270 \mathrm{~km} \mathrm{~s}^{-1}$ for accreting VLM stars and BDs. However, this cutoff can discard VLM objects with very low accretion rates as discussed in Jayawardhana et al. (2003). Consequently, for young VLM stars and BDs, Jayawardhana et al. (2003) proposed $v_{10}>200 \mathrm{~km} \mathrm{~s}^{-1}$ for accretors. In this paper, we used the Jayawardhana et al. (2003) indicator of accretion. For the spectra of DENIS1538-1038, the $v_{10}$ values all are above $240 \mathrm{~km} \mathrm{~s}^{-1}$ (Table 2). This confirms that the BD undergoes accretion. Using the $v_{10}$ versus $\dot{M}$ relation in Natta et al. (2004), we estimated mass accretion rates of $10^{-7.9}-10^{-10.5} M_{\odot} \mathrm{yr}^{-1}$. The accretion rates in the M5.5 dwarf observed on 12 Aug. 2017 and 17 Jul. 2018 are comparable to those measured in the two M5 dwarfs in Scorpius-Centarus (Murphy et al. 2015), but they are $>1.4$ orders of magnitude more intense on the remaining dates, implying accretion bursts.

Here, it is important to discuss the possibilities that the $\mathrm{H} \alpha$ variability observed in DENIS1538-1038 could be due to variable accretion (e.g., Scholz \& Jayawardhana 2006) or flares as seen in LP 655-48 (Phan-Bao et al. 2017). Typically, the [O I] $6300 \AA$ forbidden emission is attributed to outflow associated with accretion, while $\mathrm{H} \alpha$ emission can be associated with accretion or with flares. Therefore, in accretion-driven outflow (e.g., Cabrit et al. 1990) [O I] 6300 Å forbidden emission should be correlated with the $\mathrm{H} \alpha$ emission. In DENIS1538-1038, our EW measurements of the [O I] emission line (see Fig. 9) as well as Na I D1 and D2 (Table 2) show a slight decrease with decreasing $v_{10}$ of the $\mathrm{H} \alpha$ profile. This suggests that the $\mathrm{H} \alpha$ variability is due to variable accretion, not flaring activity. We therefore conclude that the accretion in the source is sporadic. With an age of $\sim 1 \mathrm{Myr}$, a mass of $\sim 47 M_{J}$, and accretion rates of $10^{-7.9}-10^{-10.5} M_{\odot} \mathrm{yr}^{-1}$, DENIS1538-1038 is one of the youngest $\mathrm{M} /$ brown dwarfs yet observed to exhibit sporadic (Lawson et al. 2004; Scholz \& Jayawardhana 2006; Jayawardhana et al. 2006; Murphy et al. 2011, 2015, 2018) and very intense accretion (Comerón et al. 2003, 2010; White \& Basri 2003; Muzerolle et al. 2003, 2005; Jayawardhana et al. 2003; Natta et al. 2004; Herczeg \& Hillenbrand 2008; Manara et al. 2015; Santamaría-Miranda et al. 2018). Sporadic accretion at very early stages with low accretion rates prevents VLM cores (e.g., Dang-Duc et al. 2016) from accreting enough material to become stars (Phan-Bao et al. 2014).

For DENIS1538-1039, the $v_{10}$ values of all spectra are below the cutoff of $200 \mathrm{~km} \mathrm{~s}^{-1}$. We therefore classify the M3.5 dwarf as nonaccretor. Figure 10 shows the observed profiles of $\mathrm{H} \alpha$ emission in the dwarf.

\subsection{Accretion disk emission}

Based on the photometric data, the Gaia parallax of DENIS1538-1038, and the BT-Settl models with solar metallicity as mentioned in Sect. 2, our SED (see Fig. 2) yields a best-fit BT-Settl model of $T_{\star}=2800 \pm 100 \mathrm{~K}$ with $\log g=3.5 \pm 0.5$, and a blackbody with $T_{\text {disk }}=224 \mathrm{~K}$ and $L_{\mathrm{IR}} / L_{\star}=0.05$. Our estimated $T_{\star}$ value is relevant for an M5.5 \pm 0.5 dwarf at $1 \mathrm{Myr}$ when compared to a well-measured value of $\sim 2700 \pm 100 \mathrm{~K}$ for an M6.5 $\pm 0.5 \mathrm{BD}$ at a similar age (Stassun et al. 2007).

Our model SED poorly fits the $W 2$ data point as seen in Fig. 2. This is possibly due to the fact that the nearby M3.5 dwarf DENIS1538-1039 is much brighter in the W2 band than DENIS1538-1038 (see Fig. 1 and Table 1). In addition, the spatial resolution of the $W 2$ image $\left(6.4^{\prime \prime}\right)$ is poorer than that of $W 1$ $\left(6.1^{\prime \prime}\right)$. Therefore, the flux measured for DENIS1538-1038 in W2 might be contaminated by DENIS1538-1039 much more than it is in $W 1$ and $W 3$. As discussed in Sect. 2, the resolution of the $W 4$ image could not spatially resolve the two M dwarfs. Therefore, the measured W4 flux of DENIS1538-1038 that might include the flux from DENIS1538-1039 could affect our SED fitting. To subtract the W4-band flux from DENIS1538-1039, we estimated the flux from the best-fit BTSettl model to the photometric data (except W4) of the source. We then reconstructed the SED of DENIS1538-1038. The revised $W 4$ flux yields a blackbody with $T_{\text {disk }}=300 \mathrm{~K}$ and $L_{\mathrm{IR}} / L_{\star}=0.04$.

\section{Summary}

In this paper, we present our detection of the accreting M5.5 dwarf, DENIS1538-1038, based on its strong IR excesses in all four WISE bands. Using the Gaia trigonometric paralax, we estimated an age of $1 \mathrm{Myr}$ and a mass of $47 M_{J}$ for DENIS1538-1038. The lithium detection in DENIS1538-1038 also confirms its youth. Based on $\mathrm{H} \alpha$ emission line profiles, we estimated the accretion rate in DENIS1538-1038 in the range of $10^{-7.9}-10^{-10.5} M_{\odot} \mathrm{yr}^{-1}$. These accretion rate values are generally lower than the typical values of $10^{-6}-10^{-8} M_{\odot} \mathrm{yr}^{-1}$ for young low-mass stars (e.g., Hartigan et al. 1995) by about two orders of magnitude. The observed $\mathrm{H} \alpha$ variability indicates a 
D. Nguyen-Thanh et al.: Sporadic and intense accretion in a 1 Myr-old brown dwarf

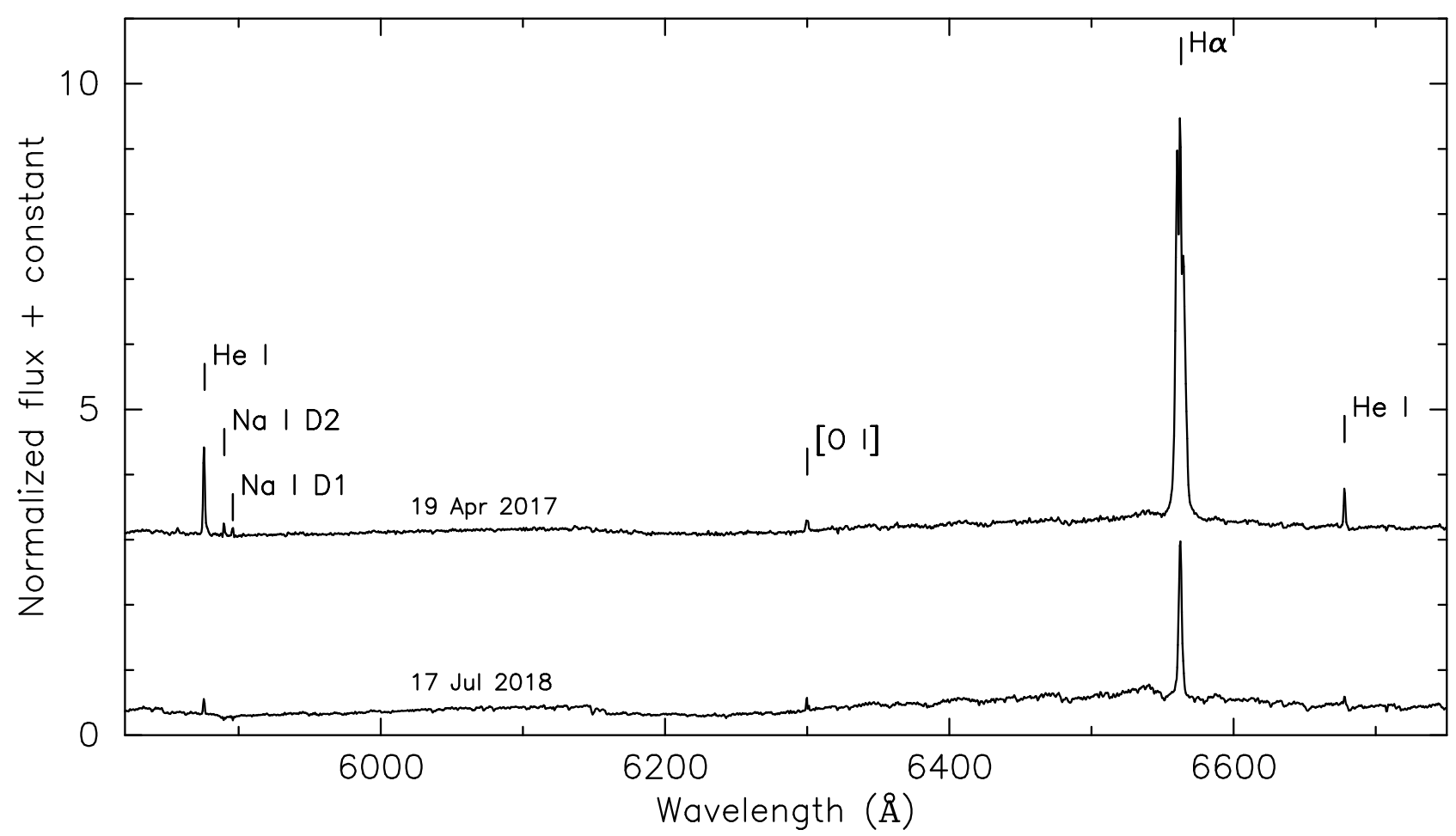

Fig. 7. Representative spectra of DENIS1538-1038 observed on Apr. 19, 2017 and Jul. 17, 2018. The H $\alpha$, He I at $5876 \AA$ and $6678 \AA$, Na I D1 $(5895.9 \AA)$ and D2 (5889.9 $\mathrm{A})$, and [O I] $6300 \AA$ lines are indicated. Strong H $\alpha$ emission that is significantly variable indicates sporadic accretion (see Sect. 3.5 for further details).

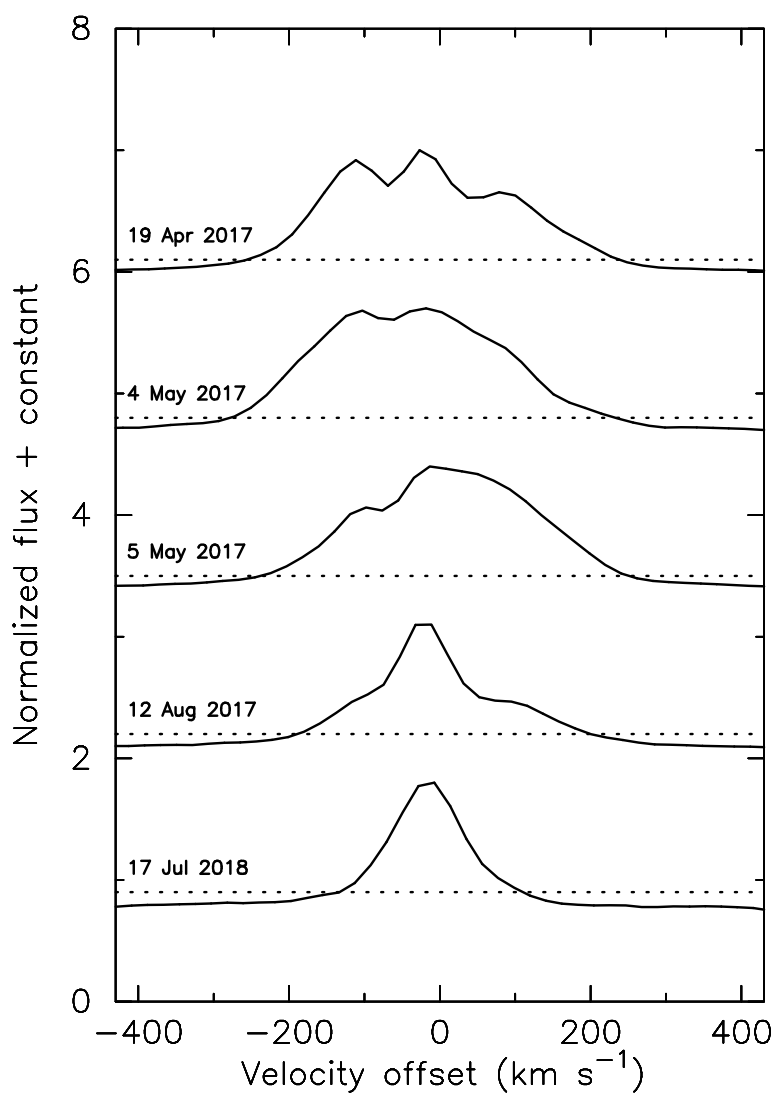

Fig. 8. $\mathrm{H} \alpha$ velocity profiles of DENIS1538-1038. The dashed line indicates the full width at $10 \%$ of the peak.

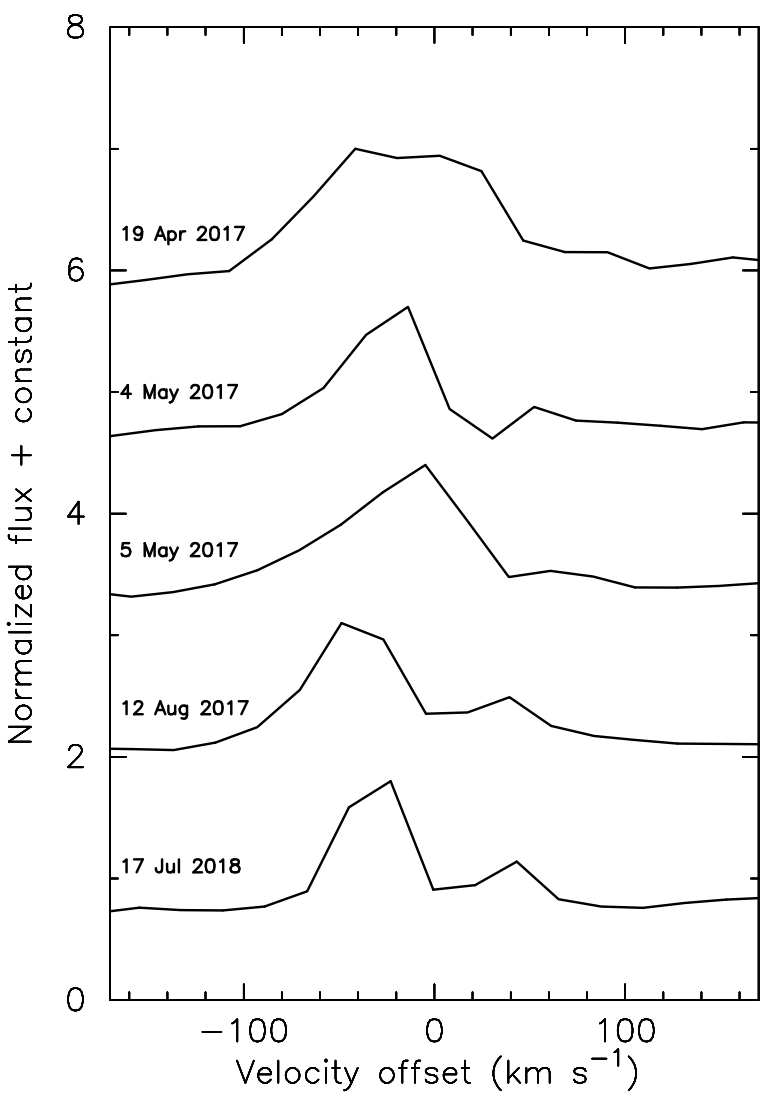

Fig. 9. [O I] $6300 \AA$ A velocity profiles of DENIS1538-1038. 


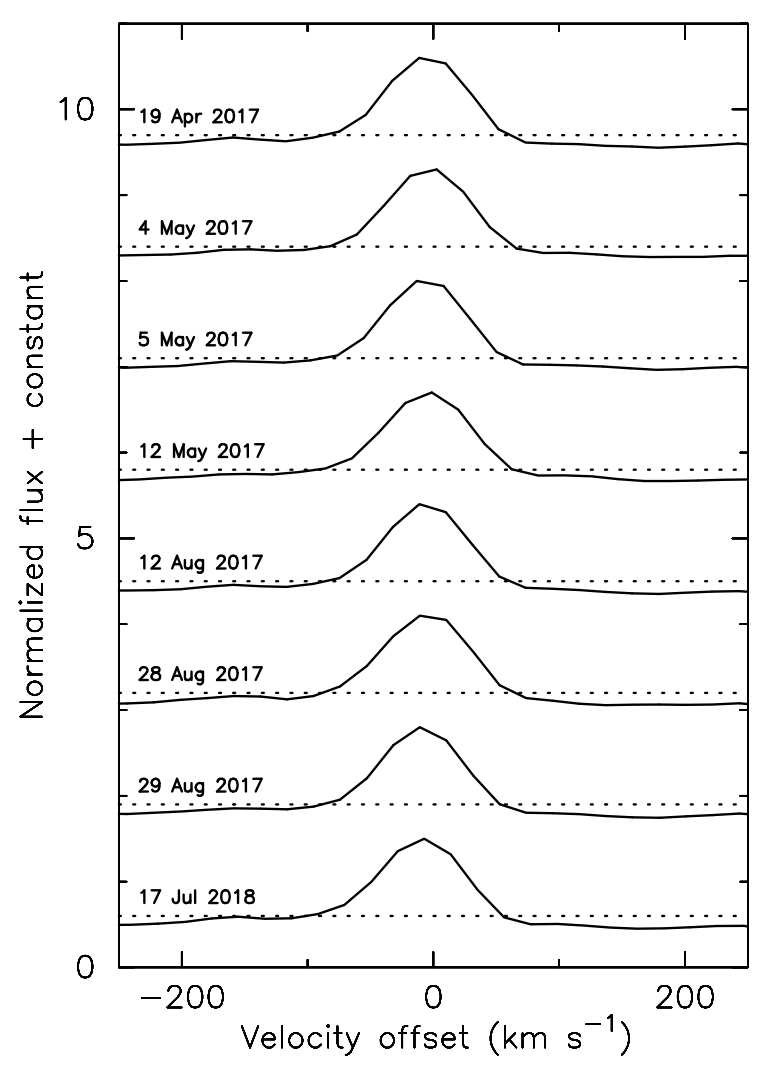

Fig. 10. Same as Fig. 8, but for DENIS1538-1039.

sporadic accretion in the 1-Myr old BD. Our discovery of the 1-Myr old BD that exhibits sporadic accretion with low accretion rates supports a possible scenario for $\mathrm{BD}$ formation (Phan-Bao et al. 2014) where low accretion rates at very early stages (possibly with high ratios of outflow to accretion rate) prevent VLM cores from accreting enough gas to become stars, and thus these cores would end up as BDs.

Acknowledgements. This research is funded by Vietnam National Foundation for Science and Technology Development (NAFOSTED) under grant number 103.99-2015.108. This publication makes use of data products from the Widefield Infrared Survey Explorer, which is a joint project of the University of California, Los Angeles, and the Jet Propulsion Laboratory/California Institute of Technology, funded by the National Aeronautics and Space Administration. This work has made use of data from the European Space Agency (ESA) mission Gaia (https://www. cosmos.esa.int/gaia), processed by the Gaia Data Processing and Analysis Consortium (DPAC, https://www. cosmos.esa.int/web/ gaia/dpac/consortium). Funding for the DPAC has been provided by national institutions, in particular the institutions participating in the Gaia Multilateral Agreement. The DENIS project has been partly funded by the SCIENCE and the HCM plans of the European Commission under grants CT920791 and CT940627. It is supported by INSU, MEN and CNRS in France, by the State of Baden-Württemberg in Germany, by DGICYT in Spain, by CNR in Italy, by FFwFBWF in Austria, by FAPESP in Brazil, by OTKA grants F-4239 and F013990 in Hungary, and by the ESO C\&EE grant A-04-046. Jean Claude Renault from IAP was the Project manager. Observations were carried out thanks to the contribution of numerous students and young scientists from all involved institutes, under the supervision of P. Fouqué, survey astronomer resident in Chile. This publication makes use of data products from the Two Micron All Sky Survey, which is a joint project of the University of Massachusetts and the Infrared Processing and Analysis Center/California Institute of Technology, funded by the National Aeronautics and Space Administration and the National Science Foundation. This research has made use of the VizieR catalogue access tool, CDS, Strasbourg, France. The original description of the VizieR service was published in Ochsenbeil et al. (2000). This research has made use of the SIMBAD database, operated at CDS, Strasbourg, France. We thank the referee, Elisabetta Rigliaco, for a useful report.

\section{References}

Allard, F., Homeier, D., Freytag, B., Schaffenberger, W., \& Rajpurohit, A. S. 2013, Mem. Soc. Astron. It., 24, 128

Avenhaus, H., Schmid, H. M., \& Meyer, M. R. 2012, A\&A, 548, A105

Basri, G. 2000, ARA\&A, 38, 485

Bessell, M. S. 1991, AJ, 101, 662

Bessell, M. S. 1999, PASP, 111, 1426

Boucher, A., Lafrenière, D., Gagné, J., et al. 2016, ApJ, 832, 50

Cabrit, S., Suzan, E., Strom, S. E., \& Strom, K. M. 1990, ApJ, 354, 687

Close, L. M., Siegler, N., Potter, D., Brandner, W., \& Liebert, J. 2002, ApJ, 567, L53

Cohen, M., Wheaton, WM. A., \& Megeath, S. T. 2003, AJ, 126, 1090

Comerón, F., Fernández, M., Baraffe, I., Neuhäuser, R., \& Kaas, A. A. 2003, A\&A, 406, 1001

Comerón, F., Testi, L., \& Natta, A. 2010, A\&A, 522, A47

Crifo, F., Phan-Bao, N., Delfosse, X., et al. 2005, A\&A, 441, 653

Cutri, R. M., et al. 2014, VizieR On-line Data Catalog: II/311

Dang-Duc, C., Phan-Bao, N., \& Dao-Van, D. T. 2016, A\&A, 588, L2

Dopita, M., Hart, J., McGregor, P., et al. 2007, Ap\&SS, 310, 255

Flaherty, K., Hughes, A. M., Mamajek, E. E., \& Murphy, S. J. 2019, ApJ, 872, 92

Fouqué, P., Chevallier, L., Cohen, M., et al. 2000, A\&AS, 141, 313

Gagné, J., Mamajek, E. E., Malo, L., et al. 2018, ApJ, 856, 23

Hartigan, P., Edwards, S., \& Ghandour, L. 1995, ApJ, 452, 736

Herczeg, G. J., \& Hillenbrand, L. A. 2008, ApJ, 681, 594

Jayawardhana, R., Mohanty, S., \& Basri, G. 2003, ApJ, 592, 282

Jayawardhana, R., Coffey, J., Scholz, A., Brandeker, A., \& Van Kerkwijk, M. H. 2006, ApJ, 648, 1206

Lawson, W. A., Lyo, A-Ran, \& Muzerolle, J. 2004, MNRAS, 351, L39

Luhman, K. L., Joergens, V., Lada, C., et al. 2007, in Protostars and Planets V, eds. B. Reipurth, D. Jewitt, \& K. Keil (Tucson, AZ: University of Arizona Press), 443

Luhman, K. L., Herrmann, K. A., Mamajek, E. E., Esplin, T. L., \& Pecault, M. J. 2018, AJ, 156, 76

Manara, C. F., Testi, L., Natta, A., \& Alcalá, J. M. 2015, A\&A, 579, A66

Martín, E. L., Phan-Bao, N., Bessell, M. S., et al. 2010, A\&A, 517, A53

Montagnier, G., Ségransan, D., Beuzit, J.-L. 2006, A\&A, 460, L19

Murphy, S. J., \& Lawson, W. A. 2015, MNRAS, 447, 1267

Murphy, S. J., Lawson, W. A., Bessell, M. S., \& Bayliss, D. D. J. 2011, MNRAS, 411, L51

Murphy, S. J., Lawson, W. A., \& Bento, J. 2015, MNRAS, 453, 2220

Murphy, S. J., Mamajek, E. E., \& Bell, C. P. M. 2018, MNRAS, 476, 3290

Muzerolle, J., Hillenbrand, L., Calvet, N., Briceño, C., \& Hartmann, L. 2003, ApJ, 592, 266

Muzerolle, J., Luhman, K. L., Briceño, C., Hartmann, L., \& Calvet, N. 2005, ApJ, 625, 906

Natta, A., Testi, L., Muzerolle, J., et al. 2004, A\&A, 424, 603

Ochsenbeil, F., Bauer, P., \& Marcout, J. 2000, A\&AS, 143, 23

Pavlenko, Y. V., Rebolo, R., Martín E. L., \& García López, R. J. 1995, A\&A, 303, 807

Pecaut, M. J., \& Mamajek, E. E. 2013, ApJS, 208, 9

Phan-Bao, N., \& Bessell, M. S. 2006, A\&A, 446, 515

Phan-Bao, N., Guibert, J., Crifo, F., et al. 2001, A\&A, 380, 590

Phan-Bao, N., Crifo, F., Delfosse, X., et al. 2003, A\&A, 401, 959

Phan-Bao, N., Forveille, T., Martín, E. L., \& Delfosse, X. 2006, ApJ, 645, L153

Phan-Bao, N., Lee, C.-F., Ho, P. T. P., Dang-Duc, C., \& Li, D. 2014, ApJ, 795, 70

Phan-Bao, N., Bessell, M. S., Nguyen-Thanh, D., et al. 2017, A\&A, 600, A19

Reid, I. N., \& Gizis, J. E. 2005, PASP, 117, 676

Reid, I. N., Cruz, K. L., Allen, P., et al. 2003, AJ, 126, 3007

Reiners, A. 2009, ApJ, 702, L119

Reiners, A., Seifahrt, A., \& Dreizler, S. 2010, A\&A, 513, L9

Rigliaco, E., Natta, A., Testi, L., Randich, S., Alcalà, J. M., et al. 2012, A\&A, 548, A56

Riedel, A. R., DiTomasso, V., Rice, E. L., et al. 2019, AJ, 157, 247

Rizzuto, A. C., Ireland, M. J., \& Kraus, A. L. 2015, MNRAS, 448, 2737

Santamaría-Miranda, A., Cáceres, C., Schreiber, M. R., et al. 2018, MNRAS, 475, 2994

Scholz, A., \& Jayawardhana, R. 2006, ApJ, 638, 1056

Shortridge, K., Meyerdierks, H., Currie, M., et al. 2004, Starlink User Note, 86

Silverberg, S. M., Kuchner, M. J., Wisniewski, J. P., et al. 2016, ApJ, 830, L28

Stassun, K. G., Mathieu, R. D., \& Valenti, J. A. 2007, ApJ, 664, 1154

White, R. J., \& Basri, G. 2003, ApJ, 582, 1109

Wright, E. L., Eisenhardt, P. R. M., Mainzer, A. K., et al. 2010, AJ, 140, 1868 
Appendix A: SED of late-M dwarfs
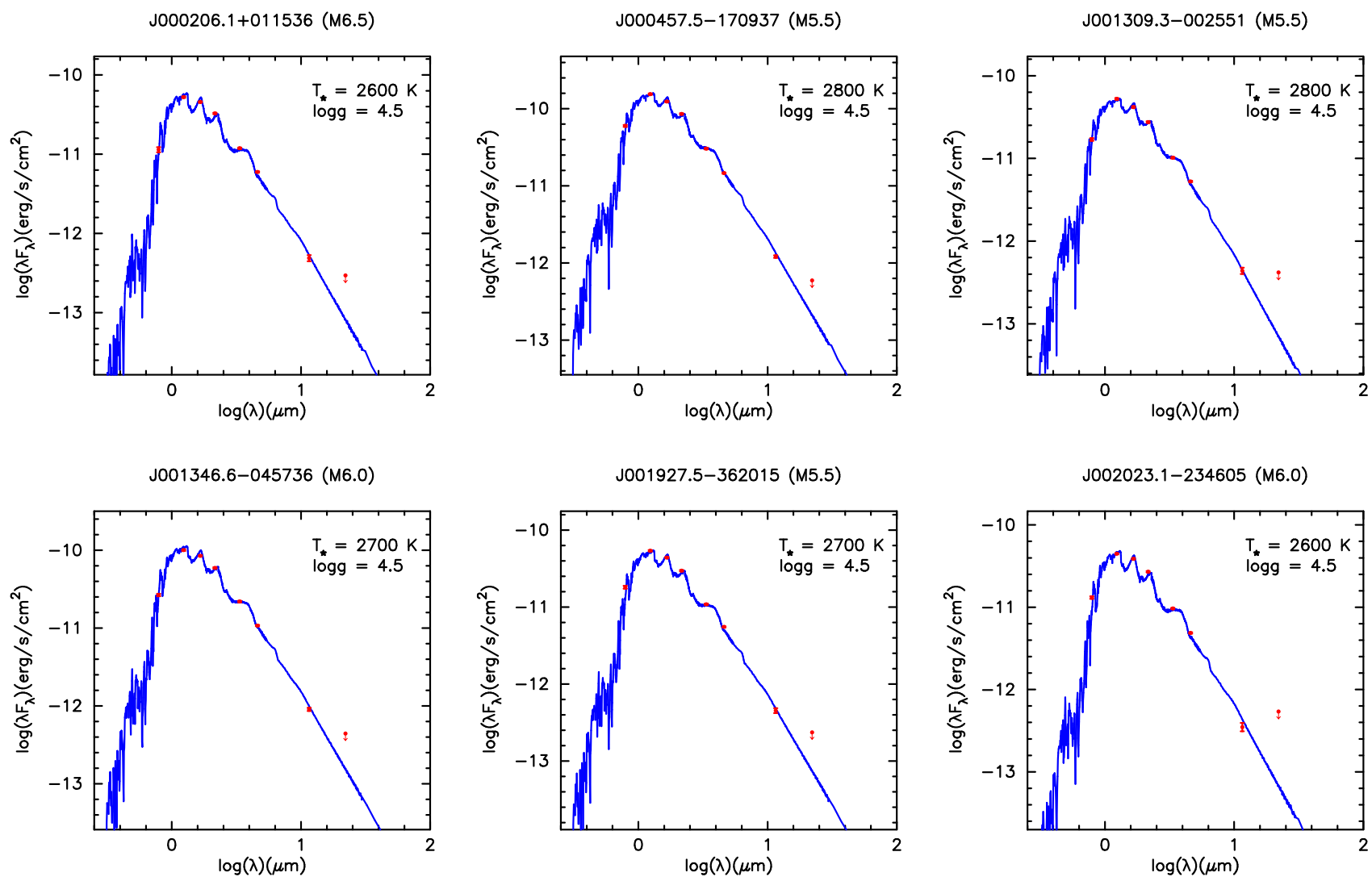

J004135.3-562112 (M6.5+M9.0)
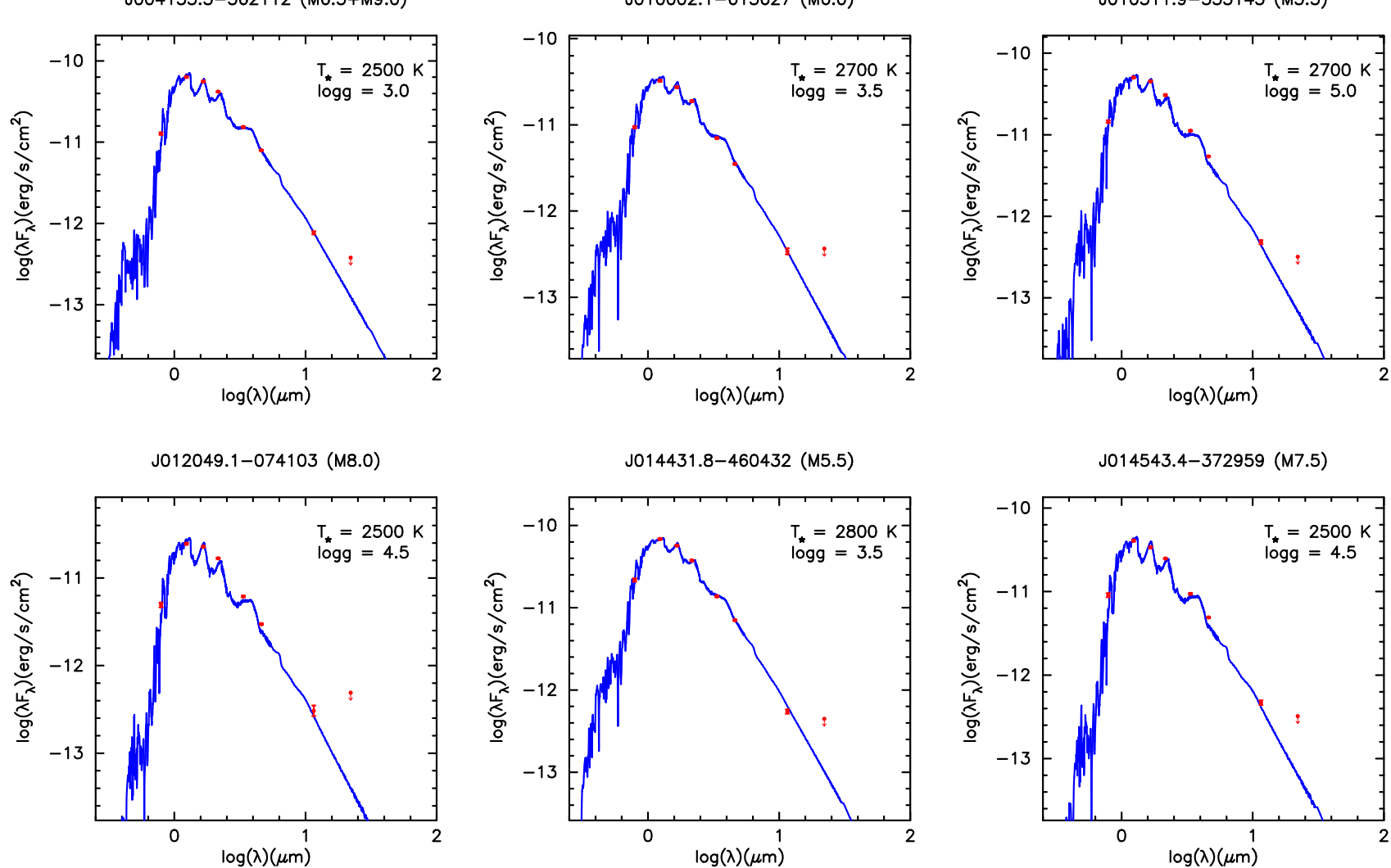

Fig. A.1. Effective temperature $T_{\star}$ and surface gravity $\log g$ from the best-fit BT-Settl model (blue solid line) are shown. Spectral types are mainly taken from Phan-Bao \& Bessell (2006), Crifo et al. (2005), Phan-Bao et al. (2017). Except J004135.3-562112, which were taken from Reiners (2009), J041048.0-125142 from Phan-Bao et al. (2006), J084818.9-201911 from Reid \& Gizis (2005), J141159.9-413221 from Phan-Bao et al. (2017) and Montagnier et al. (2006), J150416.1-235556 from Reid et al. (2003), and J220622.7-204706 from Crifo et al. (2005) and Close et al. (2002). 

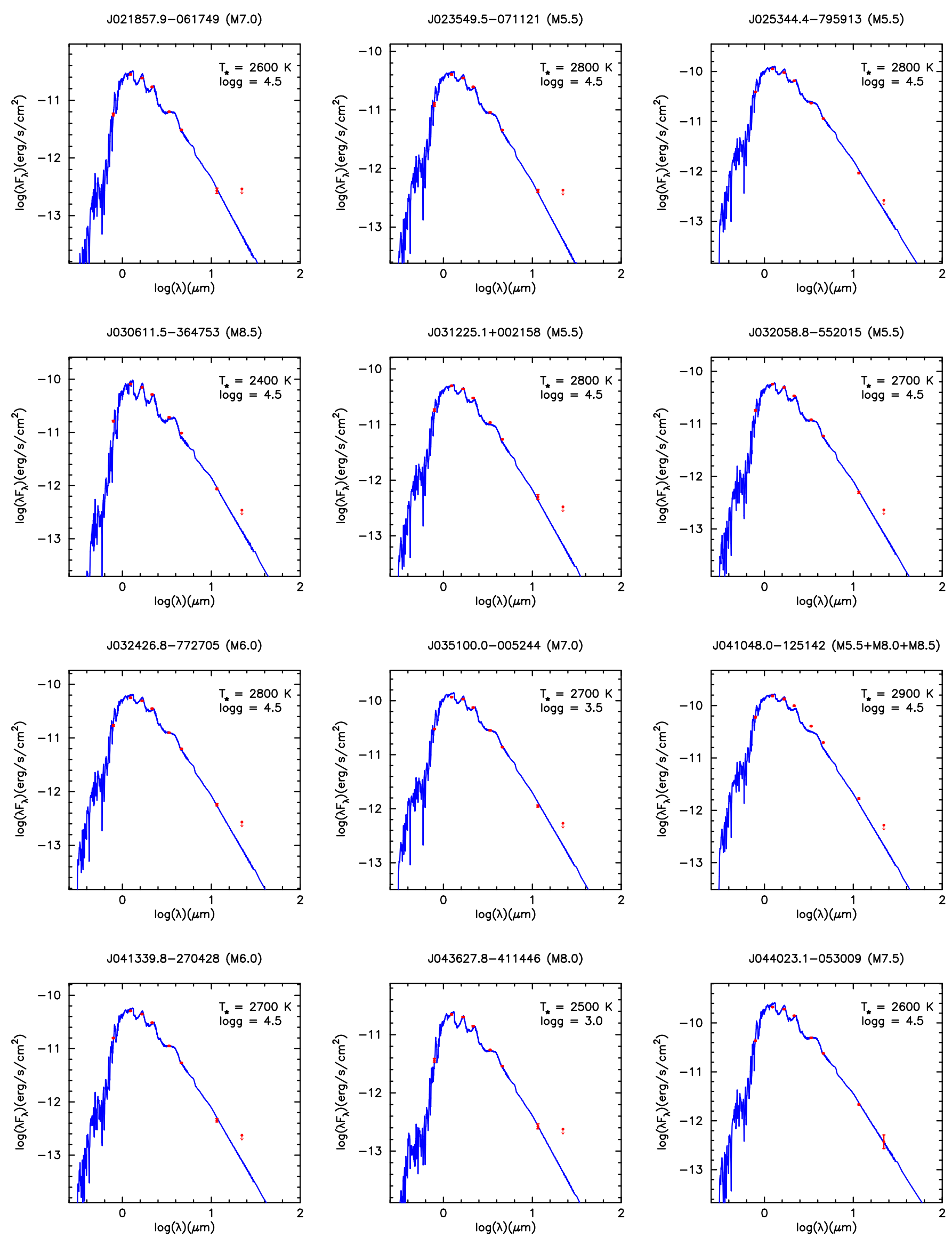

Fig. A.1. continued. 
D. Nguyen-Thanh et al.: Sporadic and intense accretion in a 1 Myr-old brown dwarf
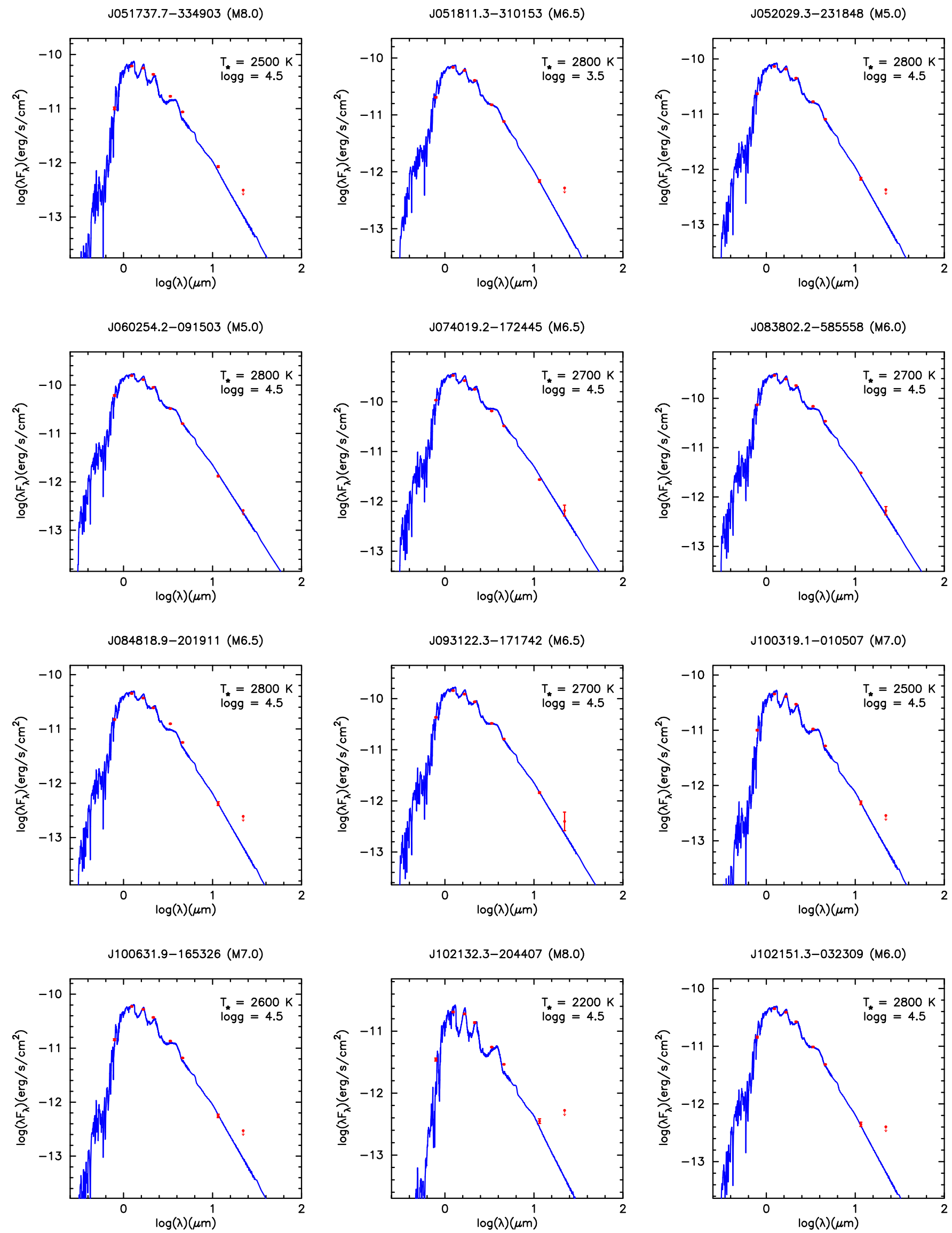

Fig. A.1. continued. 

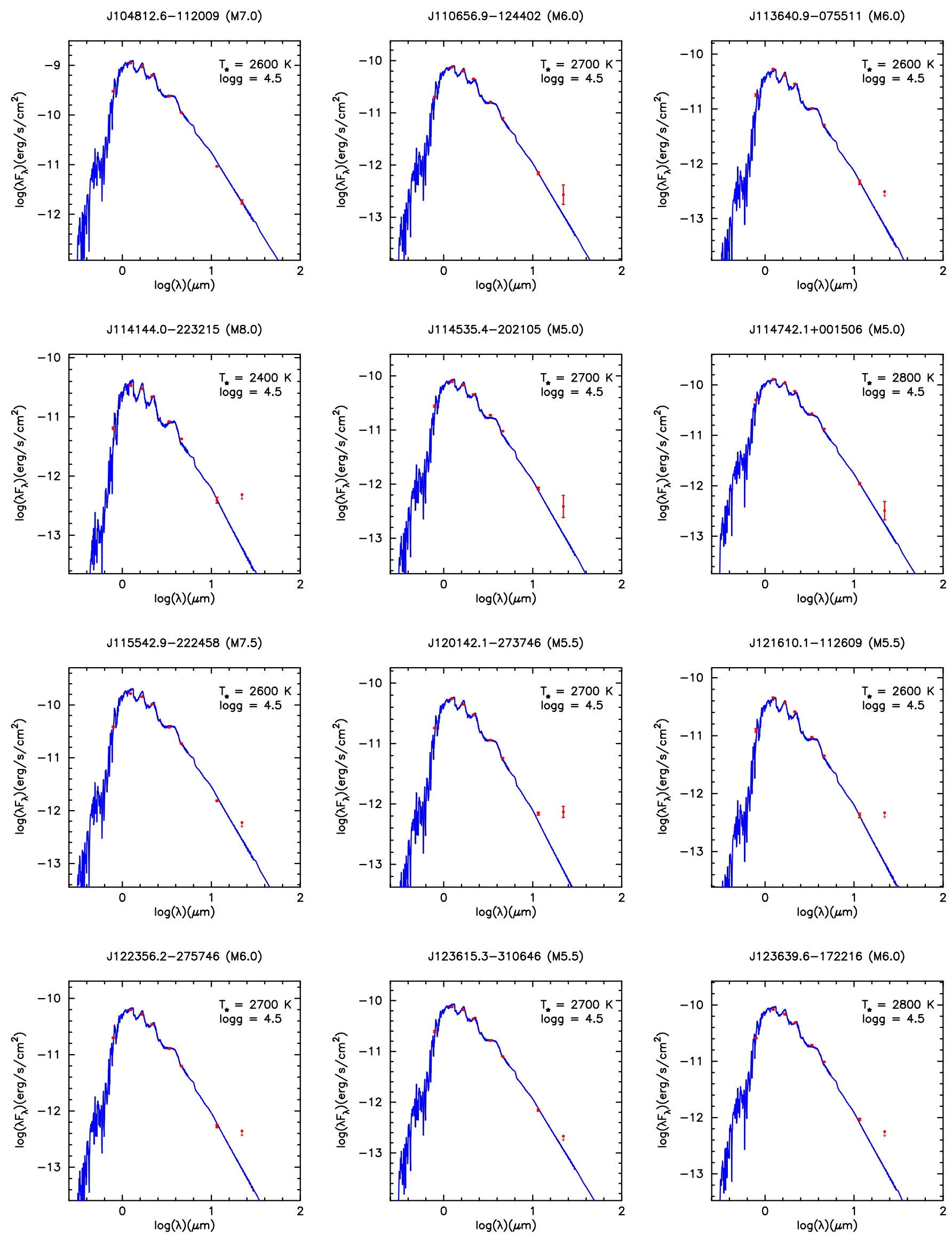

Fig. A.1. continued. 
D. Nguyen-Thanh et al.: Sporadic and intense accretion in a 1 Myr-old brown dwarf
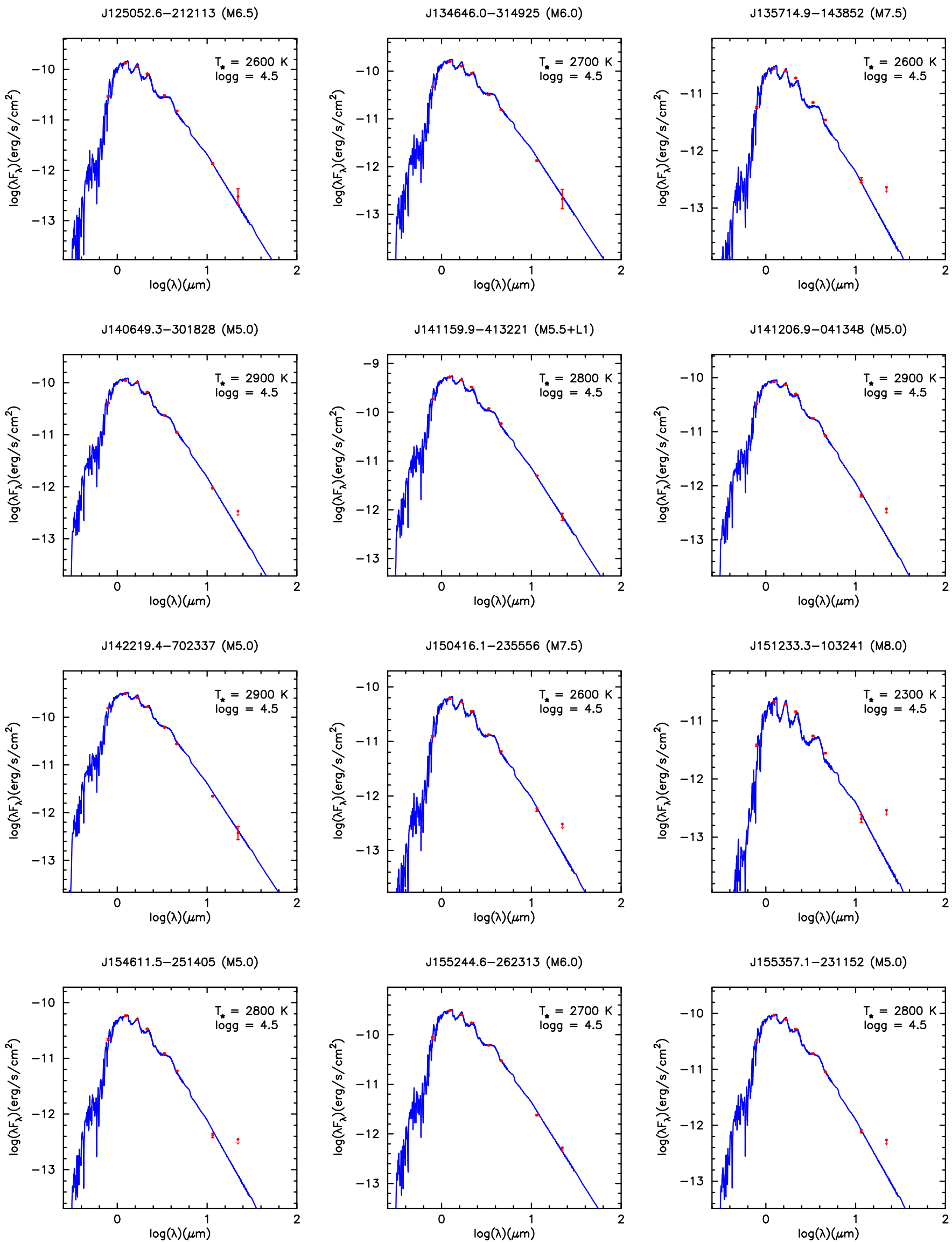

Fig. A.1. continued. 

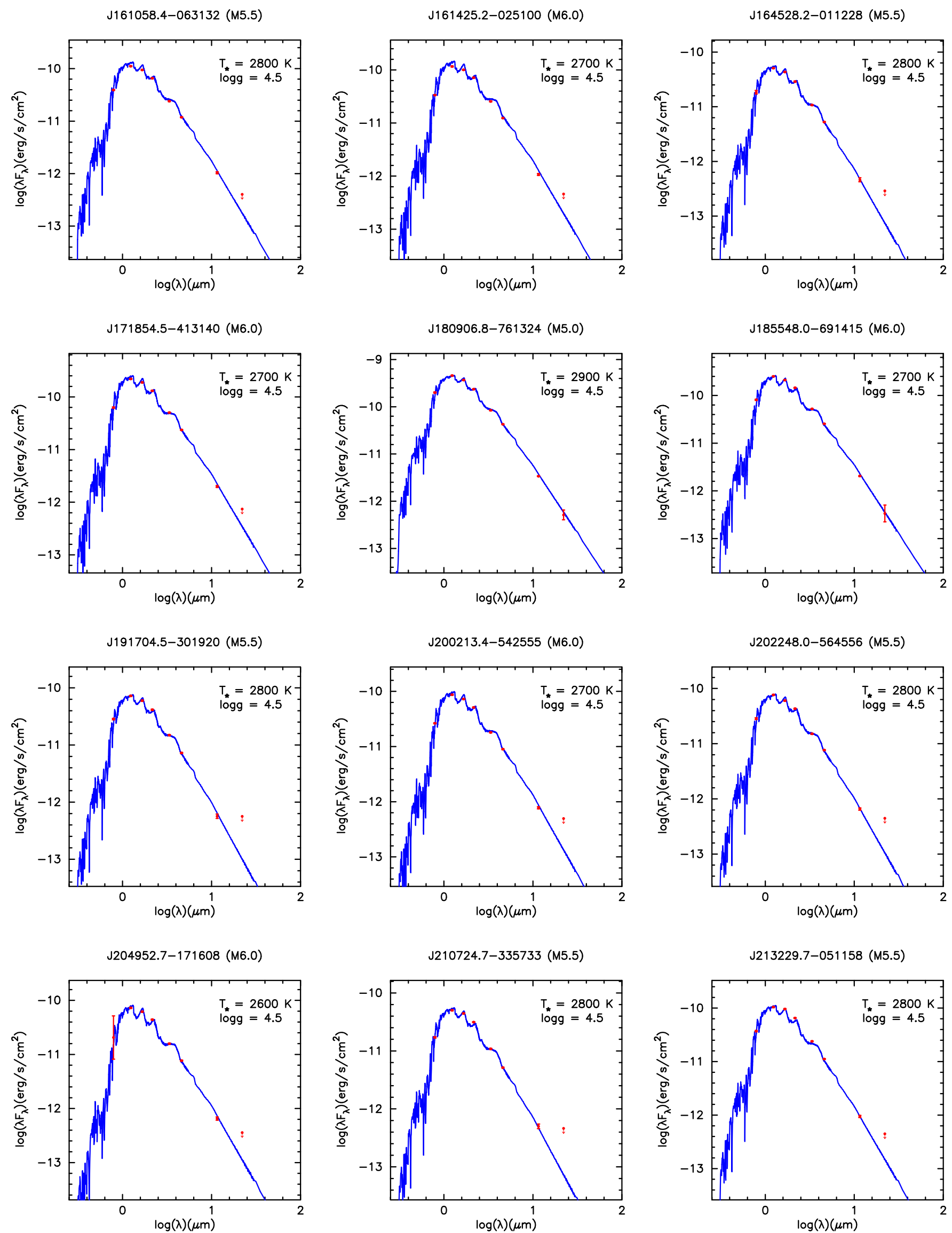

Fig. A.1. continued. 
D. Nguyen-Thanh et al.: Sporadic and intense accretion in a 1 Myr-old brown dwarf
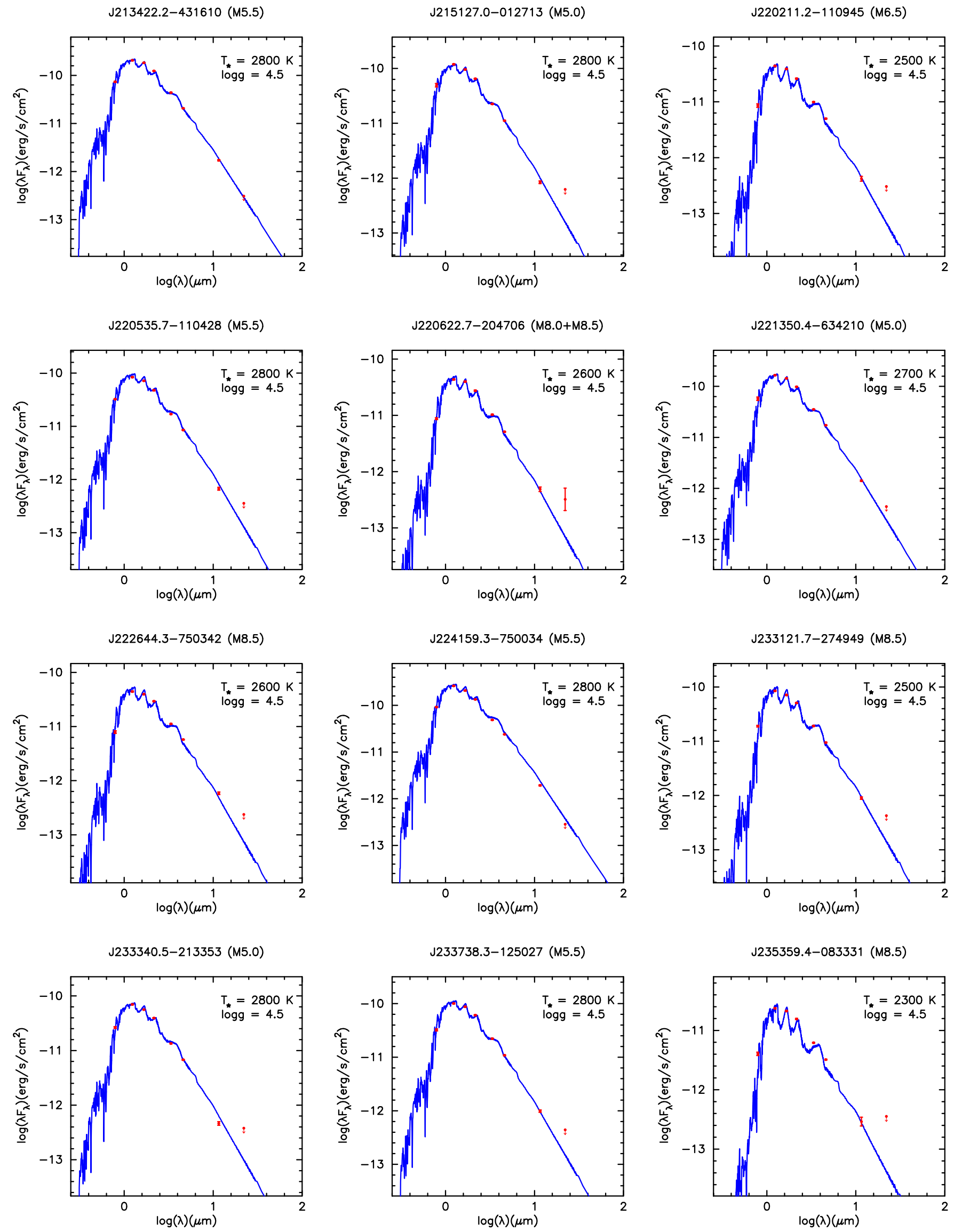

Fig. A.1. continued. 